\title{
SOME OPERATIONS ON LATTICE IMPLICATION ALGEBRAS
}

\author{
E. H. ROH, S. Y. KIM, Y. XU, and Y. B. JUN
}

(Received 16 November 2000)

\begin{abstract}
We introduce the concept of a $\otimes$-closed set and a $\otimes$-homomorphism in lattice implication algebras, and we discuss some of their properties. Next, we introduce the fuzzy implicative filter and obtain equivalent conditions. Finally, we discuss the operation $\otimes$, fuzzy filters, and fuzzy implicative filters.
\end{abstract}

2000 Mathematics Subject Classification. 03G10, 06B10, 94D05.

1. Introduction. In order to research the logical system whose propositional value is given in a lattice from the semantic viewpoint, $\mathrm{Xu}$ [3] proposed the concept of lattice implication algebras, and discussed some of their properties in [3, 4]. Xu and Qin [5] introduced the notion of filters in a lattice implication algebra, and investigated their properties. In [8], Xu and Qin defined the fuzzy filter in a lattice implication algebra, and they discussed some of their properties. Recently, Xu et al. [9] defined a congruence relation on lattice implication algebras induced by fuzzy filters and they proved the fuzzy homomorphism fundamental theorem, and Liu and $\mathrm{Xu}$ [1] introduced the notion of the new binary operation $\otimes$ on lattice implication algebras and they investigated their properties.

2. Preliminaries. We recall a few definitions and properties.

DEFINITION 2.1 (see [4]). By a lattice implication algebra we mean a bounded lattice $(L, \vee, \wedge, 0,1)$ with order-reversing involution ", " and a binary operation " $\rightarrow$ " satisfying the following axioms:

(I1) $x \rightarrow(y \rightarrow z)=y \rightarrow(x \rightarrow z)$,

(I2) $x \rightarrow x=1$,

(I3) $x \rightarrow y=y^{\prime} \rightarrow x^{\prime}$,

(I4) $x \rightarrow y=y \rightarrow x=1 \Rightarrow x=y$,

(I5) $(x \rightarrow y) \rightarrow y=(y \rightarrow x) \rightarrow x$,

(L1) $(x \vee y) \rightarrow z=(x \rightarrow z) \wedge(y \rightarrow z)$,

(L2) $(x \wedge y) \rightarrow z=(x \rightarrow z) \vee(y \rightarrow z)$,

for all $x, y, z \in L$. If ( $L, \vee, \wedge, 0,1)$ satisfies the conditions (I1), (I2), (I3), (I4), and (I5), is called a quasi lattice implication algebra.

We can define a partial ordering $\leq$ on a lattice implication algebra $L$ by $x \leq y$ if and only if $x \rightarrow y=1$.

In a lattice implication algebra $L$, the following hold: for all $x, y, z \in L$,

(1) $0 \rightarrow x=1,1 \rightarrow x=x$, and $x \rightarrow 1=1$,

(2) $x \leq y$ implies $z \rightarrow x \leq z \rightarrow y$ and $x \rightarrow z \geq y \rightarrow z$, 
(3) $(x \rightarrow y) \rightarrow((y \rightarrow z) \rightarrow(x \rightarrow z))=1$,

(4) $x \rightarrow((x \rightarrow y) \rightarrow y)=1$,

(5) $((x \rightarrow y) \rightarrow y) \rightarrow y=x \rightarrow y$.

DEFINITION 2.2 (see [7]). Let $\left(L, \vee, \wedge,,^{\prime} \rightarrow\right)$ be a lattice implication algebra. A subset $F$ of $L$ is called a filter of $L$ if it satisfies for all $x, y \in L$

(F1) $1 \in F$,

(F2) $x \in F$ and $x \rightarrow y \in F$ imply $y \in F$.

A subset $F$ of $L$ is called an implicative filter of $L$, if it satisfies (F1) and

(F3) $x \rightarrow(y \rightarrow z) \in F$ and $x \rightarrow y \in F$ imply $x \rightarrow z \in F$

for all $x, y, z \in L$.

The following propositions are clear.

Proposition 2.3. In a lattice implication algebra, every implicative filter is a filter.

Proposition 2.4. Every filter $F$ of a lattice implication algebra $L$ has the following property:

$$
x \leq y, \quad x \in F \text { imply } y \in F .
$$

DEFINITION 2.5 (see [6]). Let $\left(L, \vee, \wedge,,^{\prime} \rightarrow\right)$ be a lattice implication algebra and $S \subseteq L$. If $S$ satisfies the following condition: $S$ is called a subalgebra of $L$ if

(i) $0 \in S$,

(ii) for all $x, y \in S, x \rightarrow y \in S$.

Definition 2.6 (see [3]). Let $\left(L, \vee, \wedge,,^{\prime}, \rightarrow, \otimes, 0,1\right)$, and $\left(L^{*}, \vee, \wedge,,^{\prime} \rightarrow, \otimes, 0,1\right)$ be lattice implication algebras and $f: L \rightarrow L^{*}$ be a mapping. Then $f$ is called the implication homomorphism if for all $x, y \in L$,

$$
f(x \rightarrow y)=f(x) \longrightarrow f(y)
$$

and $f$ is called the lattice implication homomorphism if for all $x, y \in L$,

$$
f(x \vee y)=f(x) \vee f(y), \quad f(x \wedge y)=f(x) \wedge f(y), \quad f\left(x^{\prime}\right)=f(x)^{\prime} .
$$

LEMMA 2.7 (see [3]). Let $\left(L, \vee, \wedge,,^{\prime}, \rightarrow, 0,1\right)$ and $\left(L^{*}, \vee, \wedge,,^{\prime} \rightarrow, 0,1\right)$ be lattice implication algebras. If $f: L \rightarrow L^{*}$ is a function. Then $f$ is a lattice implication homomorphism if and only if $f$ is an implication homomorphism and $f\left(a^{\prime}\right)=f(a)^{\prime}$ for all $a \in L$.

DEFINITION 2.8 (see [1]). Let $\left(L, \vee, \wedge,,^{\prime}, \rightarrow, 0,1\right)$ be a quasi-lattice implication algebra and given elements $a, b$ of $L$, we define

$$
A(a, b):=\{x \in L \mid a \leq b \rightarrow x\}
$$

If for all $x, y \in L, A(x, y)$ has a least element, written $x \otimes y$, then the quasi-lattice implication algebra is said to be with property $(P)$.

LEMMA 2.9 (see [1]). Any lattice implication algebra is with property (P), in fact $a \otimes b$ $=\left(a \rightarrow b^{\prime}\right)^{\prime}$. 
LEMMA 2.10 (see [1]). Let $\left(L, \vee, \wedge,,^{\prime} \rightarrow, 0,1\right)$ be a lattice implication algebra. Then the following hold: for all $a, b, c \in L$,

(6) $a \otimes b \leq a \wedge b \leq a$,

(7) $a \leq b$ if and only if $a \otimes b^{\prime}=0$,

(8) $a \otimes b=b \otimes a$,

(9) $(a \rightarrow b) \otimes a \leq b$,

(10) $(a \otimes b) \rightarrow c=b \rightarrow(a \rightarrow c)$.

We now review some fuzzy logic concepts. Let $X$ be a set. A function $\mu: X \rightarrow[0,1]$ is called a fuzzy subset on $X$.

In the following, it will be convenient to write simply $L$ for $\left(L, \vee, \wedge,,^{\prime} \rightarrow, \otimes, 0,1\right)$, and $L^{*}$ for $\left(L^{*}, \vee, \wedge,,^{\prime} \rightarrow, \otimes, 0,1\right)$.

3. $\otimes$-closed sets and $\otimes$-homomorphisms. In this section, we introduce the concept of a $\otimes$-closed set and a $\otimes$-homomorphism in lattice implication algebras, and we discuss their properties. If $L$ is a lattice implication algebra, then by Lemma 2.9, we know that $a \otimes b \in L$ for all $a, b \in L$. Now we define the $\otimes$-closed sets as follows.

DEFINITION 3.1. Let $L$ be a lattice implication algebra and $A$ a nonempty subset of $L$. Then $A$ is said to be $\otimes$-closed of $L$ if $a \otimes b \in A$ whenever $a, b \in A$.

THEOREM 3.2. Let $F$ be a filter of a lattice implication algebra $L$. Then $F$ is $a \otimes$-closed set in $L$.

Proof. Let $F$ be a filter of $L$ and $a, b \in F$. Then by Proposition 2.4, we have $b \rightarrow$ $(a \otimes b) \in F$, and so we get $a \otimes b \in F$. Therefore $F$ is $\otimes$-closed in $L$.

THEOREM 3.3. In a lattice implication algebra, every subalgebra is $\otimes$-closed.

Proof. Let $S$ be a subalgebra of a lattice implication algebra $L$ and $a, b \in S$. Then, since $x^{\prime}=x \rightarrow 0 \in S$ for all $x \in S$, we have

$$
a \otimes b=\left(a \longrightarrow b^{\prime}\right)^{\prime} \in S,
$$

and so $S$ is $\otimes$-closed in $L$.

REMARK 3.4. The converse of Theorems 3.2 and 3.3 are not true, and so we know that $\otimes$-closed sets are the generalization of subalgebras and filters in a lattice implication algebra.

EXAMPLE 3.5. Let $L:=\{0, a, b, c, 1\}$. Define the partially ordered relation on $L$ as $0<a<b<c<1$, and define $x \wedge y:=\min \{x, y\}, x \vee y:=\max \{x, y\}$ for all $x, y \in L$, and ", "and " $\rightarrow$ " as follows:

\begin{tabular}{|l|l|}
\hline$x$ & $x^{\prime}$ \\
\hline 0 & 1 \\
\hline$a$ & $c$ \\
\hline$b$ & $b$ \\
\hline$c$ & $a$ \\
\hline 1 & 0 \\
\hline
\end{tabular}

\begin{tabular}{|c|ccccc|}
\hline$\rightarrow$ & 0 & $a$ & $b$ & $c$ & 1 \\
\hline 0 & 1 & 1 & 1 & 1 & 1 \\
\hline$a$ & $c$ & 1 & 1 & 1 & 1 \\
\hline$b$ & $b$ & $c$ & 1 & 1 & 1 \\
\hline$c$ & $a$ & $b$ & $c$ & 1 & 1 \\
\hline 1 & 0 & $a$ & $b$ & $c$ & 1 \\
\hline
\end{tabular}


Then $\left(L, \vee, \wedge,,^{\prime} \rightarrow\right)$ is a lattice implication algebra and we can find the following $\otimes$-table:

\begin{tabular}{|c|ccccc|}
\hline$\otimes$ & 0 & $a$ & $b$ & $c$ & 1 \\
\hline 0 & 0 & 0 & 0 & 0 & 0 \\
\hline$a$ & 0 & 0 & 0 & 0 & $a$ \\
\hline$b$ & 0 & 0 & 0 & $a$ & $b$ \\
\hline$c$ & 0 & 0 & $a$ & $b$ & $c$ \\
\hline 1 & 0 & $a$ & $b$ & $c$ & 1 \\
\hline
\end{tabular}

Now we consider the $\otimes$-closed set $A:=\{0, a, b\}$, which is neither a filter nor a subalgebra of $L$ since $0 \in A$ and $b \rightarrow a=c \notin A$.

DeFinITION 3.6. Let $L$ and $L^{*}$ be lattice implication algebras and let $f: L \rightarrow L^{*}$ be a function. Then $F$ is called a $\otimes$-homomorphism if for all $a, b \in L$,

$$
f(a \otimes b)=f(a) \otimes f(b) .
$$

THEOREM 3.7. Let $f: L \rightarrow L^{*}$ be a $\otimes$-homomorphism of lattice implication algebras. If $f^{-1}(0):=\{a \in L \mid f(a)=0\} \neq \varnothing$, then $f^{-1}(0)$ is $\otimes$-closed in $L$.

Proof. Let $a, b \in f^{-1}(0)$. Then we have $f(a \otimes b)=f(a) \otimes f(b)=0 \otimes 0=0$, and so $a \otimes b \in f^{-1}$, that is, $f^{-1}(0)$ is a $\otimes$-closed set in $L$.

We discuss the connection between lattice implication homomorphisms and $\otimes$ homomorphisms.

THEOREM 3.8. Let $f: L \rightarrow L^{*}$ be a function of lattice implication algebras. If $f$ is a lattice implication homomorphism, then $f$ is a $\otimes$-homomorphism.

Proof. For any $a, b \in L$, we have

$$
f(a \otimes b)=f\left(\left(a \longrightarrow b^{\prime}\right)^{\prime}\right)=f\left(a \longrightarrow b^{\prime}\right)^{\prime}=\left(f(a) \longrightarrow f(b)^{\prime}\right)^{\prime}=f(a) \otimes f(b),
$$

and hence $f$ is a $\otimes$-homomorphism.

We consider the converse of Theorem 3.8, so we have the following theorem.

THEOREM 3.9. Let $f: L \rightarrow L^{*}$ be a function of lattice implication algebras. If $f$ is $a \otimes$-homomorphism and $f\left(a^{\prime}\right)=f(a)^{\prime}$ for all $a \in L$, then $f$ is a lattice implication homomorphism.

Proof. For any $a, b \in L$, we have

$$
f(a \longrightarrow b)=f\left(\left(a \otimes b^{\prime}\right)^{\prime}\right)=f(a) \longrightarrow f(b) .
$$

Thus by Lemma 2.7, we obtain that $f$ is a lattice implication homomorphism.

By Theorems 3.8 and 3.9, we have the following corollary. 
COROLLARY 3.10. Let $f: L \rightarrow L^{*}$ be a function of lattice implication algebras. Then $f$ is a lattice implication homomorphism if and only if $f$ is $a \otimes$-homomorphism and $f\left(a^{\prime}\right)=f(a)^{\prime}$ for all $a \in L$.

THEOREM 3.11. Let $L$ and $L^{*}$ be lattice implication algebras, and $f$ and $g$ be functions from $L$ into $L^{*}$. If $f(a \otimes b)=g(a \otimes b)$ for all $a, b \in L$, then $f=g$.

Proof. For any $x \in L$, we have $f(x)=f(x \otimes 1)=g(x \otimes 1)=g(x)$, and so $f=g$.

THEOREM 3.12. Let $f: L \rightarrow L^{*}$ be a surjective $\otimes$-homomorphism of lattice implication algebras. If $A$ is $\otimes$-closed in $L$, then $f(A)$ is $\otimes$-closed in $L^{*}$.

Proof. For any $a, b \in f(A)$, there exist $x, y$ in $A$ such that $f(x)=a$ and $f(y)=b$. Thus we have $a \otimes b=f(x) \otimes f(y)=f(x \otimes y)$. Since $A$ is $\otimes$-closed in $L$, we get $a \otimes b \in$ $f(A)$, and hence $f(A)$ is $\otimes$-closed in $L^{*}$.

THEOREM 3.13. Let $f: L \rightarrow L^{*}$ be a $\otimes$-homomorphism of lattice implication algebras. If $M^{*}$ is $\otimes$-closed in $L^{*}$, then $f^{-1}\left(M^{*}\right)$ is $\otimes$-closed in $L$.

Proof. Suppose that $M^{*}$ is $\otimes$-closed in $L^{*}$ and let $a, b \in f^{-1}\left(M^{*}\right)$. Then we have $f(a) \in M^{*}$ and $f(b) \in M^{*}$. Since $M^{*}$ is $\otimes$-closed in $L^{*}$, we get $f(a \otimes b)=f(a) \otimes f(b) \in M^{*}$, and so $a \otimes b \in f^{-1}\left(M^{*}\right)$, that is, $f^{-1}\left(M^{*}\right)$ is $\otimes$-closed in $L$.

4. The operation $\otimes$ and the fuzzy (implicative) filters. In this section, we introduce the fuzzy implicative filter and obtain equivalent conditions of implicative filters. Moreover, we discuss the operation $\otimes$, fuzzy filters and fuzzy implicative filters.

DEFINITION 4.1 (see [8]). A fuzzy subset $\mu$ of a lattice implication algebra $L$ is called a fuzzy filter if it satisfies for any $x, y \in L$,

(FF1) $\mu(1) \geq \mu(x)$,

(FF2) $\mu(y) \geq \min \{\mu(x \rightarrow y), \mu(x)\}$.

Proposition 4.2 (see [8]). Let $\mu$ be a fuzzy filter of a lattice implication algebra $L$ and $x, y \in L$. Then $x \leq y$ implies $\mu(x) \leq \mu(y)$.

THEOREM 4.3. If $\mu$ is a fuzzy filter of a lattice implication algebra $L$, then for any $x, y \in L$,

(FF3) $\mu(x \otimes y) \geq \min \{\mu(x), \mu(y)\}$.

Proof. Let $\mu$ be a fuzzy filter of $L$. Then for all $x, y \in L$, we have

$$
\mu(x \otimes y) \geq \min \{\mu(y \longrightarrow(x \otimes y)), \mu(y)\} \geq \min \{\mu(x), \mu(y)\} .
$$

Let $\mu$ be a fuzzy filter of a lattice implication algebra $L$ and $x, y \in L$. Then, using Proposition 4.2 and (FF3), we have $\mu(1) \geq \mu(x)$ and

$$
\mu(y) \geq \mu(x \wedge y)=\mu(x \otimes(x \rightarrow y)) \geq \min \{\mu(x), \mu(x \rightarrow y)\}
$$

Thus by Theorem 4.3, we have the following theorem. 
THEOREM 4.4. Let $L$ be a lattice implication algebra. A fuzzy set $\mu$ in $L$ is a fuzzy filter if and only if $\mu$ is an order preserving set and (FF3) holds.

We provide an equivalent condition for a fuzzy set to be a fuzzy filter.

THEOREM 4.5. Let $L$ be a lattice implication algebra. A fuzzy set $\mu$ in $L$ is a fuzzy filter if and only if it satisfies for all $x, y, z \in L$,

(FF4) $x \geq y \otimes z$ implies $\mu(x) \geq \min \{\mu(y), \mu(z)\}$.

Proof. Suppose that $\mu$ is a fuzzy filter of $L$ and let $x, y, z \in L$ be such that $x \geq$ $y \otimes z$. Then by Theorem 4.3, we have

$$
\begin{aligned}
\mu(x) & \geq \min \{\mu((y \otimes z) \longrightarrow x), \mu(y \otimes z)\} \\
& =\min \{\mu(1), \mu(y \otimes z)\} \\
& \geq \min \{\mu(y), \mu(z)\}
\end{aligned}
$$

and so (FF4) holds.

Conversely, suppose that $\mu$ satisfies (FF4). Since $1 \geq x \otimes x$ for all $x \in L$, it follows from (FF4) that

$$
\mu(1) \geq \min \{\mu(x), \mu(x)\}=\mu(x) \quad \forall x \in L .
$$

Note that $(y \rightarrow x) \otimes y \leq x$ for all $x, y \in L$. Hence, by (FF4), we have $\mu(x) \geq$ $\min \{\mu(y \rightarrow x), \mu(y)\}$, which proves (FF2). Therefore $\mu$ is a fuzzy filter of $L$.

Now, we give an equivalent condition of implicative filters as follows.

LEMMA 4.6 (see [2]). Let $F$ be an implicative filter of $L$. Then for any $x, y \in L$,

$$
(x \rightarrow y) \longrightarrow x \in F \text { implies } x \in F .
$$

THEOREM 4.7. A nonempty subset $F$ of $L$ is an implicative filter if and only if it satisfies (F1) and

(F4) $z \rightarrow((x \rightarrow y) \rightarrow x) \in F$ and $z \in F$ imply $x \in F$ for all $x, y, z \in L$.

Proof. Suppose that $F$ satisfies (F1) and (F4). Let $x, z \in L$ be such that $z \rightarrow x \in F$ and $z \in F$. In (F4), we take $y=x$, then

$$
z \longrightarrow((x \longrightarrow x) \longrightarrow x)=z \longrightarrow(1 \longrightarrow x)=z \longrightarrow x \in F
$$

Thus by (F4), we have $x \in F$. This says that $F$ is a filter of $L$. Let $x, y, z \in L$ be such that $z \rightarrow(y \rightarrow x) \in F$ and $z \rightarrow y \in F$. Since $z \rightarrow(y \rightarrow x)=y \rightarrow(z \rightarrow x) \leq(z \rightarrow$ $y) \rightarrow(z \rightarrow(z \rightarrow x))$, we have $z \rightarrow(z \rightarrow x) \in F$. As $((z \rightarrow x) \rightarrow x) \rightarrow(z \rightarrow x)=z \rightarrow$ $(((z \rightarrow x) \rightarrow x) \rightarrow x)=z \rightarrow(z \rightarrow x) \in F$, it follows that $1 \rightarrow(((z \rightarrow x) \rightarrow x) \rightarrow$ $(z \rightarrow x)) \in F$. Combining (F1) and (F4) we obtain $z \rightarrow x \in F$. This means that $F$ is an implicative filter of $L$. 
Conversely, suppose that $F$ is an implicative filter of $L$. Let $x, y, z \in L$ be such that $z \rightarrow((x \rightarrow y) \rightarrow x) \in F$ and $z \in F$. Then by Proposition 2.4, we have $(x \rightarrow y) \rightarrow x \in F$. By Lemma 4.6, we get $x \in F$.

We state the fuzzification of implicative filters.

DEFINITION 4.8. A fuzzy subset $\mu$ of a lattice implication algebra $L$ is called a fuzzy implicative filter if it satisfies (FF1) and

(FF5) $\mu(x) \geq \min \{\mu(z \rightarrow((x \rightarrow y) \rightarrow x)), \mu(z)\}$ for all $x, y, z \in L$.

THEOREM 4.9. In a lattice implication algebra, every fuzzy implicative filter is a fuzzy filter.

Proof. In (FF5), we take $y=x$, then we have

$$
\begin{aligned}
\mu(x) & \geq \min \{\mu(z \longrightarrow((x \rightarrow x) \longrightarrow x)), \mu(z)\} \\
& =\min \{\mu(z \longrightarrow(1 \longrightarrow x)), \mu(z)\} \\
& =\min \{\mu(z \longrightarrow x), \mu(z)\} .
\end{aligned}
$$

This means that $F$ is a filter of $L$.

We state an equivalent condition for a fuzzy set to be a fuzzy implicative filter.

THEOREM 4.10. Let $\mu$ be a fuzzy filter of a lattice implication algebra L. Then the following are equivalent:

(i) $\mu$ is a fuzzy implicative filter.

(ii) $\mu(x) \geq \mu((x \rightarrow y) \rightarrow x)$ for all $x, y \in L$.

(iii) $\mu(x)=\mu((x \rightarrow y) \rightarrow x)$ for all $x, y \in L$.

Proof. (i) $\Rightarrow$ (ii). Let $\mu$ be a fuzzy implicative filter of $L$. Then by (FF5), we have

$$
\mu(x) \geq \min \{\mu(1 \longrightarrow((x \longrightarrow y) \longrightarrow x)), \mu(1)\}=\mu((x \longrightarrow y) \longrightarrow x)
$$

for all $x, y \in L$. Hence the condition (ii) holds.

(ii) $\Rightarrow$ (iii). Observe that $x \leq(x \rightarrow y) \rightarrow x$ for all $x, y \in L$. Then, by Proposition 2.4, we have $\mu(x) \leq \mu((x \rightarrow y) \rightarrow x)$. It follows from (ii) that $\mu(x)=\mu((x \rightarrow y) \rightarrow x)$. Hence the condition (ii) holds.

(iii) $\Rightarrow$ (i). Suppose that the condition (iii) holds. Since $\mu$ is a fuzzy filter, by (FF2) we have

$$
\mu((x \longrightarrow y) \longrightarrow x) \geq \min \{\mu(z \longrightarrow((x \longrightarrow y) \longrightarrow x)), \mu(z)\} .
$$

Combining (iii) we obtain

$$
\mu(x) \geq \min \{\mu(z \longrightarrow((x \longrightarrow y) \longrightarrow x)), \mu(z)\},
$$

thus $\mu$ satisfies (FF5). Therefore $\mu$ is a fuzzy implicative filter of $L$.

THEOREM 4.11. Let $L$ be a lattice implication algebra. A fuzzy set $\mu$ in $L$ is a fuzzy implicative filter if and only if it satisfies

(FF6) $z \otimes u \leq(x \rightarrow y) \rightarrow x$ in L implies $\mu(x) \geq \min \{\mu(z), \mu(u)\}$ for all $x, y, z, u \in L$, 
Proof. Suppose that $\mu$ is a fuzzy implicative filter of $L$ and let $x, y, z, u \in L$ be such that $z \otimes u \leq(x \rightarrow y) \rightarrow x$. Since $\mu$ is a fuzzy filter of $L$ by Theorem 4.9, it follows from Theorems 4.5 and 4.10 that

$$
\mu(x)=\mu((x \rightarrow y) \rightarrow x) \geq \min \{\mu(z), \mu(u)\} .
$$

Conversely, suppose that $\mu$ satisfies (FF6). Obviously, $\mu$ satisfies (FF1). Since ( $z \rightarrow$ $((x \rightarrow y) \rightarrow x)) \otimes z \leq(x \rightarrow y) \rightarrow x$, (FF6) implies that

$$
\mu(x) \geq \min \{\mu(z \longrightarrow((x \longrightarrow y) \longrightarrow x)), \mu(z)\},
$$

which proves (FF5). Therefore $\mu$ is a fuzzy implicative filter of $L$.

ACKNOWLEDGEMENTS. This work was done during the first author's stay at the Southwest Jiaotong University, China. The first author is highly grateful to the Department of Applied Mathematics and the Center for Intelligent Control Development of Southwest Jiaotong University for their support.

\section{REFERENCES}

[1] J. Liu and Y. Xu, On the property (P) of lattice implication algebra, J. Lanzhou Univ. 32 (1996), 344-348.

[2] E. H. Roh, Y. Xu, and K. Qin, On implicative filters of lattice implication algebra, submitted to Bull. Korean Math. Soc.

[3] Y. Xu, Homomorphisms in lattice implication algebras, Proceedings of 5th Symposium on Multiple Valued Logic of China, 1992, pp. 206-211.

[4] _ Lattice implication algebras, J. Southwest Jiaotong Univ. 1 (1993), 20-27.

[5] Y. Xu and K. Y. Qin, Lattice H implication algebras and implication algebra classes, J. Hebei Mining and Civil Engineering Institute (1992), no. 3, 139-143.

[6] _ Lattice properties in lattice implication algebras, Collected Works on Applied Mathematics, Chengdu University of Science and Technology Press, 1992, pp. 45-48.

[7] _ On filters of lattice implication algebras, J. Fuzzy Math. 1 (1993), no. 2, 251-260. MR 94b:06016. Zbl 787.06009.

[8] _ Fuzzy lattice implication algebras, J. Southwest Jiaotong Univ. 30 (1995), no. 2, 121-127. Zbl 830.03030.

[9] Y. Xu, E. H. Roh, and K. Y. Qin, On quotient lattice implication algebras induced by fuzzy filters, submitted to J. Fuzzy Math.

E. H. Roh: Department of Mathematics Education, Chinju National University of EducATION, CHInJu 660-756, KoREA

E-mail address: ehroh@cue.ac.kr

S. Y. Kim: Department of Mathematics Education, Chinju National University of EDUCATION, CHINJU 660-756, KOREA

Y. Xu: Department of ApPlied Mathematics, SOUThWest JiaOtong University, Chengdu, Sichuan 610031, ChinA

Y. B. JUN: DePARTMENT Of MATHEMATICS EDUCATION, GYEONGSANG NATIONAL UNIVERSiTy, CHINJU 660-701, KOREA

E-mail address: ybjun@nongae.gsnu.ac.kr 


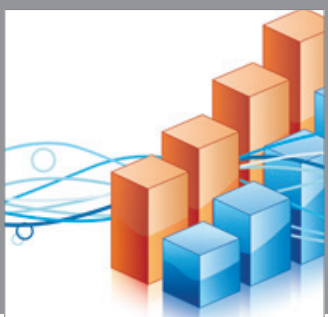

Advances in

Operations Research

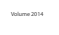

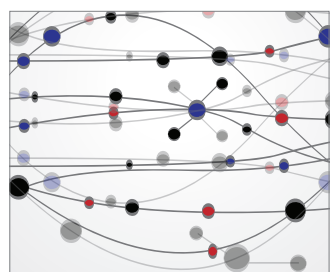

\section{The Scientific} World Journal
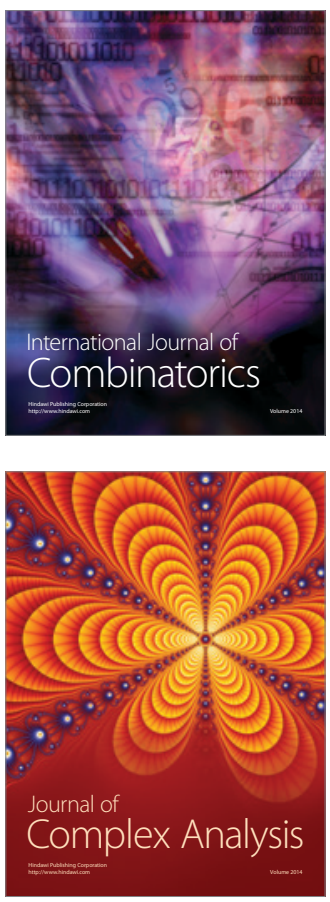

International Journal of

Mathematics and

Mathematical

Sciences
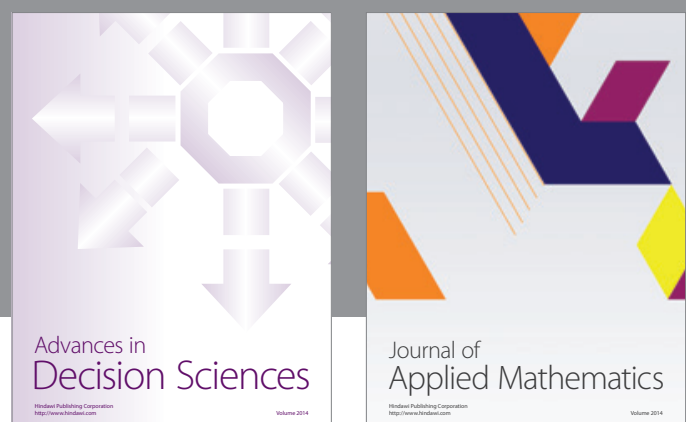

Journal of

Applied Mathematics
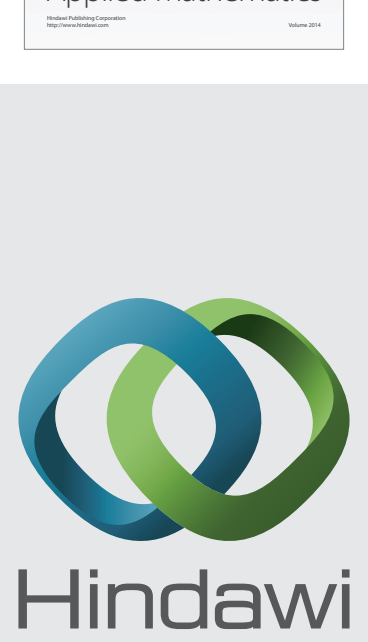

Submit your manuscripts at http://www.hindawi.com
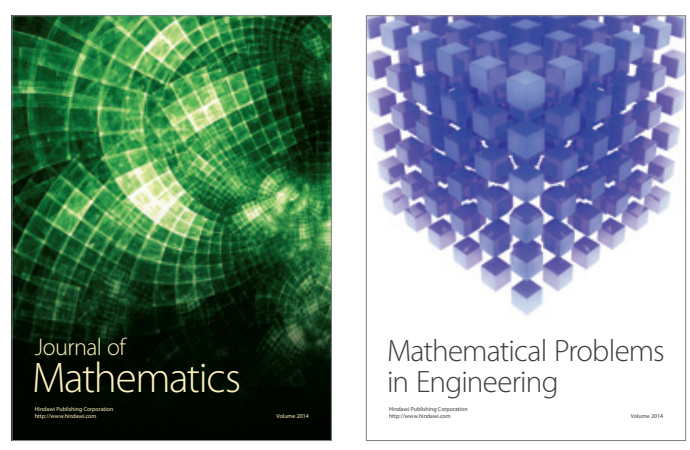

Mathematical Problems in Engineering
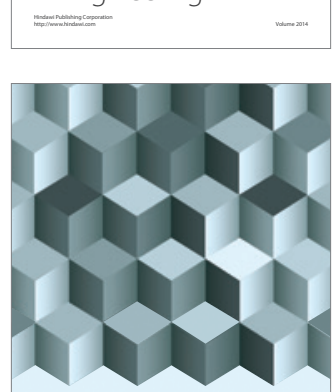

Journal of

Function Spaces
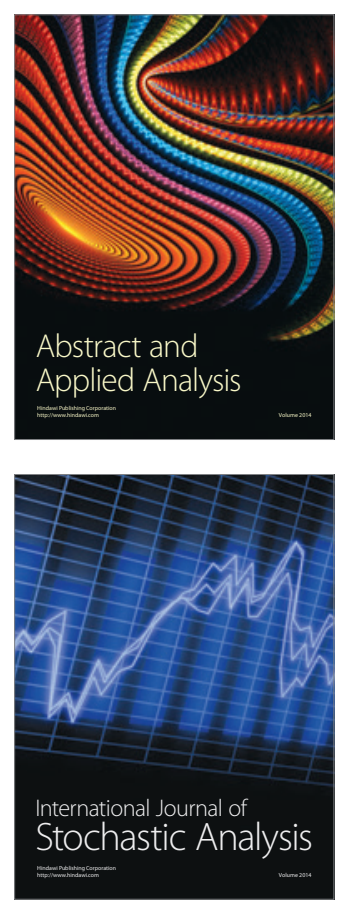

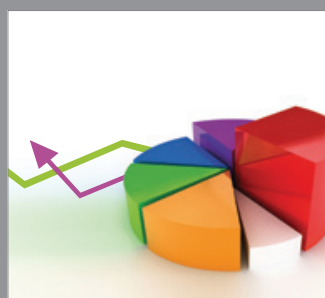

ournal of

Probability and Statistics

Promensencen
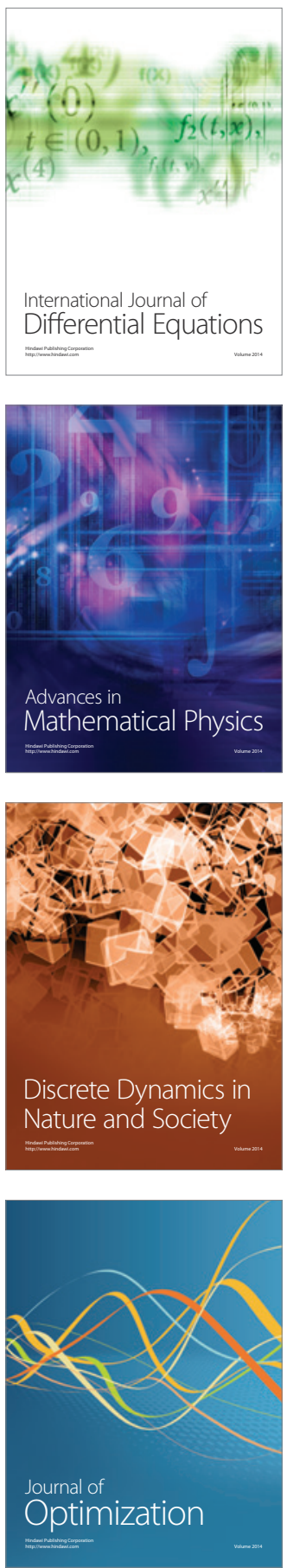